
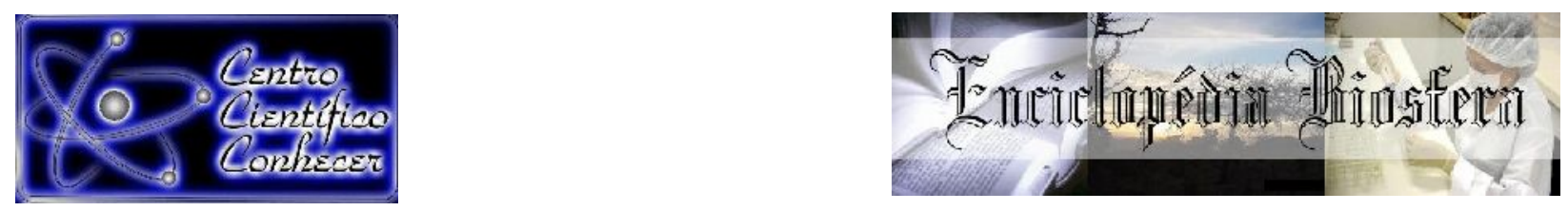

\title{
A PRÁTICA DO INSOURCING NAS ORGANIZAÇÕES
}

Euclides Fernandes dos Reis'; Sara da Costa Fernandes²; Márcio do Carmo Boareto ${ }^{3}$; Vagner Rosalem ${ }^{4}$ Vanessa Bitencourth dos Santos ${ }^{5}$.

${ }^{1}$ Mestrando em Gestão Organizacional pela Universidade Federal de Catalão (UFCAT), Catalão, Goiás (euclides.reis@yahoo.com.br).

${ }^{2}$ Mestre em Gestão Organizacional pela Universidade Federal de Goiás (UFG), Catalão, Goiás.

${ }^{3}$ Mestrando em Gestão Organizacional pela Universidade Federal de Catalão (UFCAT), Catalão, Goiás.

${ }^{4}$ Prof. Doutor na Universidade Federal de Catalão (UFCAT), Catalão, Goiás.

${ }^{5}$ Mestranda em Gestão Organizacional pela Universidade Federal de Catalão (UFCAT), Catalão, Goiás.

Recebido em: 22/09/2018 - Aprovado em: 23/11/2018 - Publicado em: 03/12/2018 DOl: 10.18677/EnciBio_2018B110

Revisão Integrativa da Literatura possui o objetivo de sistematizar o conhecimento produzido acerca dos motivos que contribuem para que as organizações revisem suas estratégias operacionais e considerem a prática do insourcing de suas atividades produtivas. Método: Levantamento realizado nas bases de dados, Web of Science, COMputerized ENgineering inDEX (COMPENDEX), Scientific Eletronic Library Online (Scielo) e ScienceDirect com os descritores: "Insourcing and Strategy and Costs", pois estes trazem maior relevância para a respectiva pesquisa. A pesquisa utilizou os idiomas inglês, espanhol e português, entre os anos de 2005 e 2018. Resultados: seis artigos atenderam aos critérios de inclusão, houve predominância de estudos de caso. Os artigos analisados descrevem o insourcing como uma possibilidade de aplicação nas organizações de diversos setores: têxtil, automobilística e tecnologia da informação com diversos objetivos estratégicos desde a redução de custos a preservação do valor estratégico da organização.

PALAVRAS-CHAVE: desterceirização, estratégia, custos.

\section{AN INSURCING PRACTICE IN ORGANIZATIONS: INTEGRATION REVIEW}

\begin{abstract}
Integrative Literature Review has the objective of systematizing the knowledge produced about the reasons that contribute to the organizations to review their operational strategies and consider the insourcing practice of their productive activities. Method: A survey carried out in the databases, Web of Science, COMputerized ENgineering inDEX (COMPENDEX), Scientific Eletronic Library Online (Scielo) e ScienceDirect with the descriptors: "Desterceirização e Estratégia e Custos", since they bring more relevance to the respective research. The research used the English, Spanish and Portuguese languages, between the years 2005 and 2018. Results: 6 articles met the inclusion criteria, there were predominance of case studies. The articles analyzed describe insourcing as a possibility of applying in the organizations of several sectors: textile, automobile and information technology with
\end{abstract}


several strategic objectives from the reduction of costs to the preservation of the strategic value of the organization.

KEYWORDS: Insourcing, Strategy, Costs.

\section{INTRODUÇÃO}

O crescimento das organizações associado às diversas mudanças tanto de cunho interno como externo, exige das organizações, maior capacidade de formular e implementar estratégias que propiciem a superação dos constantes obstáculos de mercado e atingir seus objetivos. Para Baeta et al., (2014), os desafios podem ser de ordem tecnológica, integração de mercados e redefinição do papel das organizações baseadas em um planejamento estratégico estruturado.

A crise do no modelo de produção em massa, e da transição para o modelo de produção flexível, também conhecida como produção enxuta, deu origem a uma descentralização produtiva/administrativa nas organizações. Nesta perspectiva nasceu o conceito de terceirização que se refere ao ato de transferir a responsabilidade por um determinado serviço/operação ou mesmo uma fase de um processo de produção ou de comercialização, de uma organização para outra (GIOSA, 2003).

As últimas décadas a terceirização de serviços e/ou produtos ganhou notoriedade entre as organizações sob o ponto vista estratégico, com diversos propósitos, dentre os quais se podem ressaltar a redução de custos, otimização de recursos e a dedicação exclusiva às atividades-fim (MAGALHÃES et al., 2011). Neste cenário, a "aplicação da terceirização trouxe no seu bojo uma série de mudanças estruturais, sistêmicas e comportamentais que se refletiram de forma significativa nos processos e nos resultados das organizações" (SARAIVA; MERCÊS, 2013).

A terceirização é uma técnica, onde a organização destina-se às atividades secundárias, também conhecidas como atividade-meio, do seu processo, a outra empresa especialista na atividade terceirizada. Assim a contratante se especializa no produto principal (MAGALHÃES et al., 2011). No Brasil, o método de contratar terceiros tem sido bastante utilizado pelas organizações desde o final da década de 1990. Neste contexto, baseado no estudo de Saraiva e Mercês (2013) a terceirização surgiu como forma de as empresas competirem em condições mais vantajosas e transferirem para outras empresas serviços não essenciais ao negócio.

Diante de cenários ainda mais instáveis e turbulentos as organizações são cada vez mais instigadas a superar os desafios e exigências do mercado e a partir das recentes crises vividas pela indústria no século $\mathrm{XXI}$, surgiu uma nova prática, chamada de desterceirização, também conhecida por primarização, insourcing reverse, outsorcing ou re-insorcing (DRAUZ, 2013). Ocorre o processo reverso a terceirização, no qual consiste em retomar as atividades, funções e áreas produtivas que haviam sido delegadas a empresas subcontratadas.

Assim algumas organizações mudaram suas estratégias de terceirização após a reavaliação da sua posição competitiva, criando novas condições para o desenvolvimento, fabricação, comercialização e fornecimento de serviços e/ou produtos (FREYTAG et al., 2012).

A vantagem competitiva deve ser analisada de forma desagregada e não com um enfoque generalizado dentro de uma organização, pois cada atividade contribui para custos relativos. Diante desta nova conjuntura, as organizações têm reavaliado a gestão, com o propósito de reestruturar novamente os processos, atividades bem como a estrutura funcional (PORTER, 2015). 
Sendo assim, o objetivo deste estudo foi sistematizar o conhecimento produzido acerca de como tem sido utilizada a estratégia do Insourcing nas organizações, e entender os possíveis ganhos gerados a partir deste novo conceito.

\section{MATERIAL E MÉTODOS}

Revisão Integrativa de Literatura (RI) que se fundamenta em um método de revisão específica, que através da produção científica objetiva o viés critico, bem como a síntese e evidências de outros estudos e metodologias tais como experimental, quase experimental, não experimental, qualitativo e quantitativo (MENDES et al., 2008).

A revisão integrativa oferece ao pesquisador um ponto norteador, em que se define o problema ou questão de pesquisa. Por se tratar de um assunto praticado nas organizações, mas pouco debatido na literatura científica (tem histórico de investigações com poucas publicações), busca-se verificar: Quais os motivos que impulsionaram a reconsideração do insourcing nas organizações?

A investigação ocorreu no mês de maio do ano de 2018, com a seleção de descritores para pesquisa nas bases de dados. Utilizaram-se descritores controlados: "Strategy", "Cost". Também se valeu do descritor não controlado: "Insourcing", tendo em vista que o termo "Insourcing" não consta da lista de descritores controlados em Ciências da Saúde (DeCS) da Biblioteca Virtual em Saúde (BVS), o que permitiu assim uma maior captura de artigos para a investigação. Para realização da revisão integrativa foram selecionados como bases de dados Web of Science, COMputerized ENgineering inDEX (COMPENDEX), Scientific Eletronic Library Online (Scielo) e ScienceDirect. Para esse procedimento, apenas um pesquisador procedeu com à coleta em cada base de dados.

Os critérios de inclusão na revisão integrativa foram: artigos científicos completos publicados entre os anos de 2005 e 2018, gratuitos, disponíveis online, em idioma inglês, espanhol e português que retratassem o objeto de estudo. Os textos deveriam abordar o processo de insourcing na indústria, com utilização de referenciais da literatura (acadêmica/científica) que subsidiassem a técnica e com sinalização de aplicação para um grupo específico, além de indicarem resultados práticos. Foram excluídos estudos teóricos de atualização, revisão, tese e dissertação.

A partir dos artigos que preencheram os critérios de inclusão préestabelecidos houve leitura e extração das informações de interesse. Para cada artigo da amostra final deste estudo, foi preenchido o formulário adaptado e proposto por Ursi (2005). As informações de interesse retiradas foram: autores, título do artigo, delineamento do estudo, ano de publicação, país de origem, base de dados, resultados da pesquisa original e síntese das conclusões.

Para análise do nível de evidência utilizou a classificação hierárquica proposta por Stetler et al., (1998): nível I - evidência obtida do resultado de metanálise de estudos clínicos controlados e com randomização; nível II - evidência obtida em estudo de desenho experimental; nível III - evidência obtida de pesquisas quase experimentais; nível IV- evidências obtidas de estudos não experimental, descritivos ou com abordagem metodológica qualitativa; nível $V$ - evidências obtidas de relatórios de casos ou relatos de experiências; nível VI- evidências baseadas em opiniões de especialistas ou com base em normas ou legislação. Foi realizada também uma busca manual (hand search) como estratégia de investigação nas listas da bibliografia e referências dos artigos originais com intuito de maior exploração das publicações. Os resultados da seleção estão na Figura 1. 


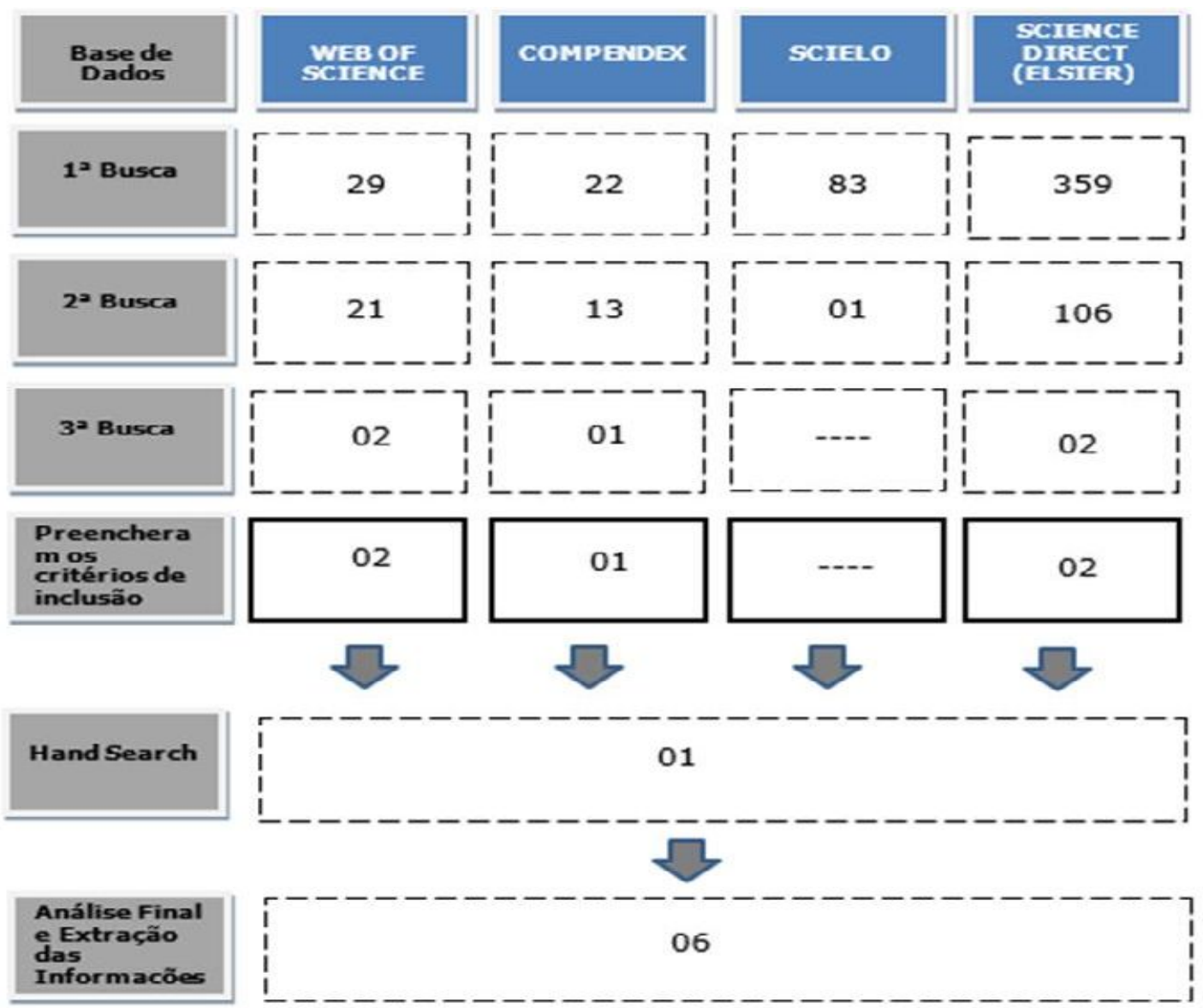

FIGURA 1 - Diagrama da coleta de dados para Revisão Integrativa sobre Insourcing at the Industry. Goiás-Brasil, 2018

Fonte: Elaborado pelos autores (2018)

\section{RESULTADOS}

A amostra desta $\mathrm{RI}$ resultou em dois artigos na Web of Science, um artigo no Compendex, dois artigos na ScienceDirect. A partir destes textos, na hand search, foi encontrado um artigo da Web of Science, totalizando seis artigos para análise final. Em números percentuais, $50,0 \%$ dos artigos estavam na Web of Science, $16,6 \%$ na Compendex e $33,3 \%$ na ScienceDirect. Na intenção de sumarizar, integralizar os achados desta $\mathrm{RI}$ e destacar as contribuições do Insourcing foi construído um quadro síntese (Quadro 1). 
QUADRO 1: Síntese dos artigos que utilizaram o Insourcing. 2005-2018.

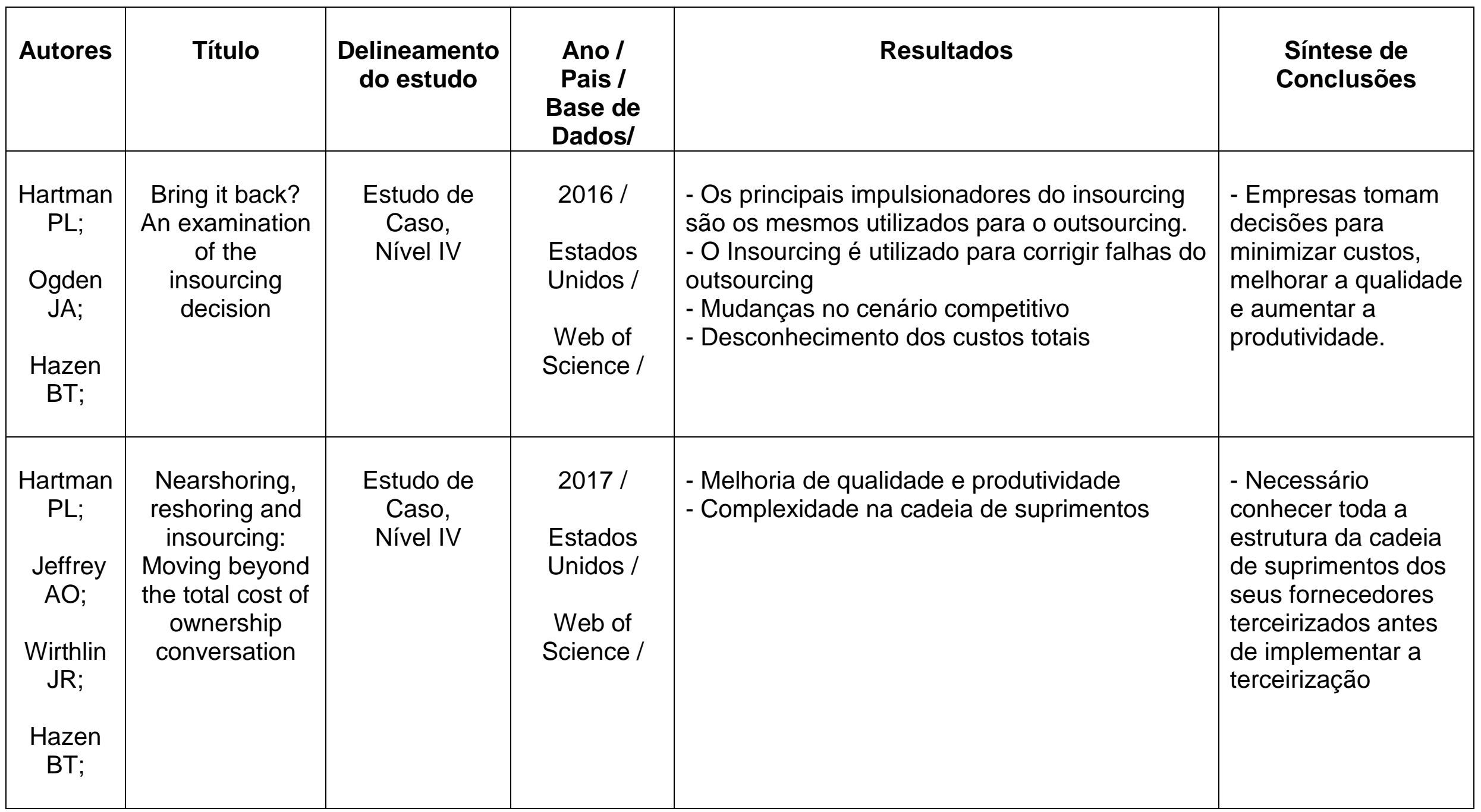




\begin{tabular}{|c|c|c|c|c|c|}
\hline Autores & Título & $\begin{array}{l}\text { Delineament } \\
\text { o do estudo }\end{array}$ & $\begin{array}{c}\text { Ano / } \\
\text { Pais / } \\
\text { Base de } \\
\text { Dados/ }\end{array}$ & Resultados & $\begin{array}{l}\text { Síntese de } \\
\text { Conclusões }\end{array}$ \\
\hline $\begin{array}{c}\text { Foerstl } \\
\text { K; } \\
\text { Kirchoff } \\
\text { JF; } \\
\text { Bals } \\
\text { L; }\end{array}$ & $\begin{array}{l}\text { Reshoring and } \\
\text { insourcing: } \\
\text { drivers and } \\
\text { future research } \\
\text { directions }\end{array}$ & $\begin{array}{c}\text { Relatório de } \\
\text { Caso, } \\
\text { Nível V }\end{array}$ & $\begin{array}{l}2016 \text { / } \\
\text { Estados } \\
\text { Unidos / } \\
\text { Web of } \\
\text { Science / }\end{array}$ & $\begin{array}{l}\text { - Redução de esforços de retrabalho. } \\
\text { - Eliminar riscos da dependência de } \\
\text { fornecedores externos } \\
\text { - Incertezas e perturbações ambientais } \\
\text { - Mudanças institucionais e politicas } \\
\text { - Complexidade da cadeia de valor } \\
\text { - Custos de comunicação elevados }\end{array}$ & $\begin{array}{l}\text { - A tecnologia é um } \\
\text { impulsionador para o } \\
\text { insourcing, pois } \\
\text { permite otimizar } \\
\text { várias etapas do } \\
\text { processo produtivo }\end{array}$ \\
\hline $\begin{array}{l}\text { Caputo } \\
\text { AC; } \\
\text { Palumb } \\
\text { o M; }\end{array}$ & $\begin{array}{l}\text { Manufacturing } \\
\text { re-insourcing } \\
\text { in } \\
\text { the textile } \\
\text { industry } \\
\text { A case study }\end{array}$ & $\begin{array}{c}\text { Estudo de } \\
\text { Caso, } \\
\text { Nível IV }\end{array}$ & $\begin{array}{c}2005 / \\
\text { Italy / } \\
\text { Compendex }\end{array}$ & - Redução de custos da produção & $\begin{array}{l}\text { - O insourcing } \\
\text { aplicado na indústria } \\
\text { têxtil possibilitou } \\
\text { flexibilidade na } \\
\text { produção e redução } \\
\text { de custos. }\end{array}$ \\
\hline
\end{tabular}




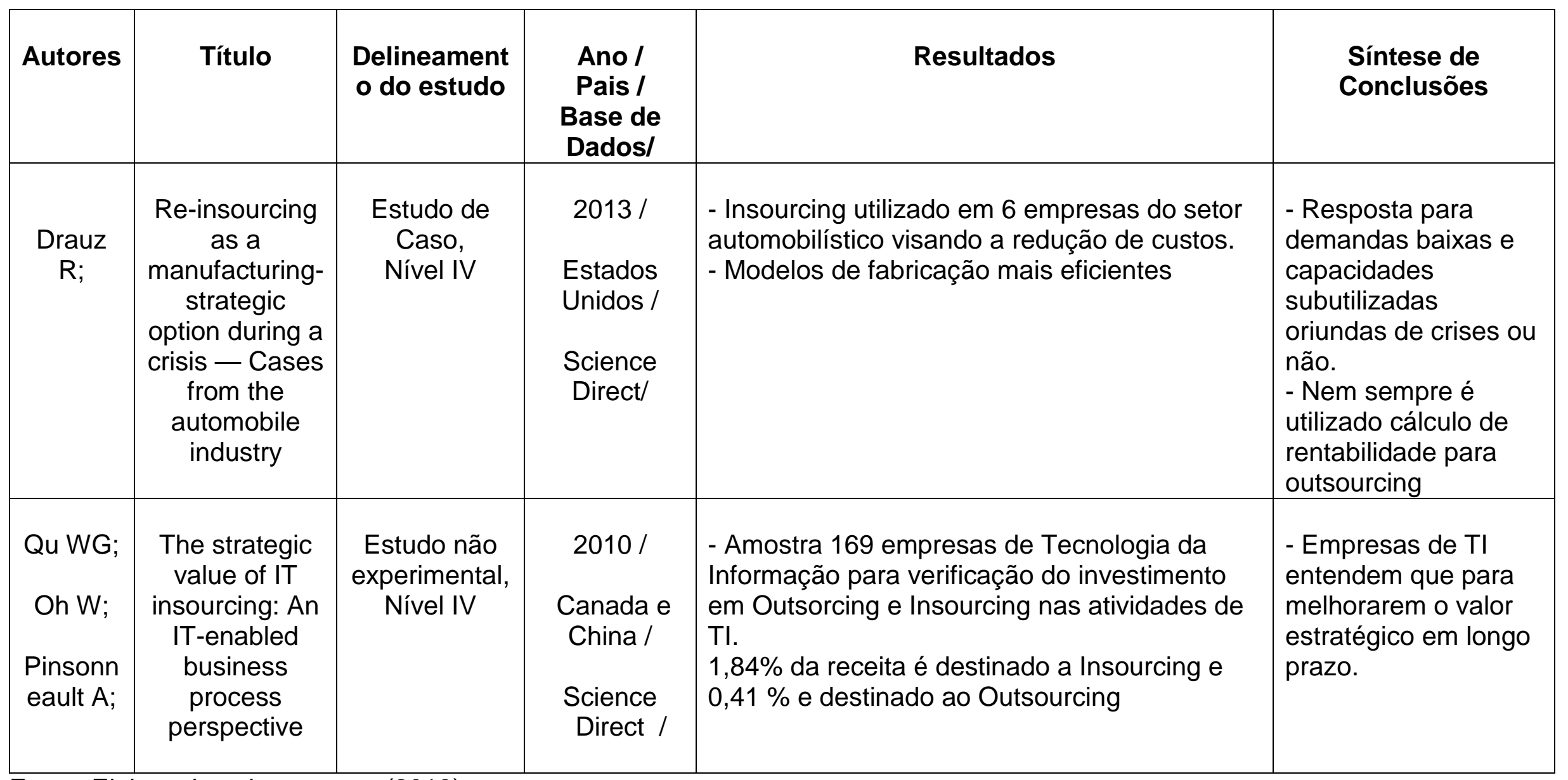

Fonte: Elaborado pelos autores (2018). 
$\mathrm{Na}$ análise dos estudos, observou-se a discussão em torno de uma prática ainda pouco aplicada, os autores retratam uma nova opção estratégica utilizada em algumas organizações diante de crises econômicas (DRAUZ, 2013). Ainda, tais estudos buscam uma reflexão sobre o momento apropriado para implementar o insourcing nas organizações (QU et al., 2010).

A abordagem do insourcing foi multiprofissional, prevalecendo publicações nas áreas administração quatro $(66,6 \%)$, engenharia, com um $(16,6 \%)$ e tecnologia da informação com um $(16,6 \%)$. Em relação ao tipo de revista científica, houve predomínio na publicação internacional com seis artigos (100,0\%), todos no idioma inglês.

Quanto à base de dados que originou os estudos, 50,0\% (HARTMAN et al., 2016; FOERSTL at al., 2016) eram provenientes da base de dados Web of Science, seguido do Compendex com 16,3\% (CAPUTO; PALUMBO, 2005), e da ScienceDirect com 33,3\% (QU et al., 2010; DRAUZ, 2013). Não houve ocorrência de duplicidade nas referidas bases de dados.

Em relação aos anos de publicação, observou-se que houve maior concentração de publicações na década de 2010, com 83,3\% (QU et al. 2010; DRAUZ, 2013; FOERSTL at al., 2016; HARTMAN et al., 2016; HARTMAN et al., 2017) e apenas $16,6 \%$ (CAPUTO; PALUMBO, 2005) anterior a esta década. Não houve publicações nos anos 2006, 2007, 2009, 2011, 2014 e 2015 de acordo com os critérios de inclusão estabelecidos nesta RI.

No que tange à origem dos artigos, observou-se que 66,66\% (DRAUZ, 2013; FOERSTL at al., 2016; HARTMAN et al., 2016; HARTMAN et al., 2017) são dos Estados Unidos; um (16,6\%) (QU et al., 2010) do Canadá; um (16,6\%) (CAPUTO; PALUMBO, 2005) da Itália. Quanto à análise do nível de evidência, os achados mostram estudos predominantes do nível IV, que se referem a estudos com metodologia não experimental ou estudos de caso, e apenas um artigo com nível de evidência VI opinião de autoridades respeitadas baseadas em sua experiência clínica, de acordo com a classificação hierárquica proposta por Stetler et al. (1998).

Os artigos analisados descrevem que em relação ao outsorcing, o insourcing pode ser uma alternativa estratégica que possibilitará a empresa em um determinado momento ser mais competitiva diante de cenários adversos (CAPUTO; PALUMBO, 2005; QU et al.2010; DRAUZ, 2013; FOERSTL at al., 2016; HARTMAN et al., 2016; HARTMAN et al., 2017).

\section{DISCUSSÃO}

Fontenelle (2014) em estudo sobre o consumo e o processo de expansão do valor, apresenta o capitalismo e o processo intensivo de crises financeiras como direcionadores a muitos estudos sobre tema, como o do sociólogo Zygmunt Bauman de 2010. Isto pode explicar porque nesta investigação foi apontado maior número publicações sobre o tema insourcing na década de 2010. Os artigos encontrados trabalham o conceito de insourcing, o qual tem se tornando em certos casos, uma estratégia contrária a pratica do outsorcing (terceirizar). $\mathrm{O}$ insourcing é a estratégia utilizada pelas organizações que retoma as atividades anteriormente exploradas internamente (SCHNIEDERJANS et al., 2015).

A principal vantagem que o insourcing traz em relação ao outsorcing "é o controle total que uma companhia alcança sobre uma atividade, devido à internalização e supervisão constante, permitindo o controle total da qualidade" (RAYCIKI, et al., 2016). Hartman et al. (2016) em estudo sobre as implicações que antecederam a estratégia de insourcing em 12 empresas da indústria manufatureira, 
afirmam que os principais fatores para insourcing nas organizações estão relacionados a eventos externos específicos de mercado e metas organizacionais de curto prazo.

A estratégia do outsorcing não foi tão produtiva quanto se imaginou ser, pois alguns custos não foram levados em consideração no momento da estratégia do outsourcing. Por esse motivo algumas organizações estão reconsiderando a estratégia insourcing para atingir os resultados: menor custo e celeridade das operações (AGUEZZOUL, 2014; HARTMAN et al., 2017).

Em um determinado estudo de caso, uma organização do setor de mineração do estado de Minas Gerais aplicou a estratégia do insourcing das atividades da área de usinagem, e constatou-se que houve redução de $24 \%$ dos custos operacionais, associado ainda a melhoria na qualidade dos serviços e ganho de produtividade (MAGALHÃES et al., 2011).

Pode-se ressaltar como benefício do insourcing, a possibilidade de assegurar o fornecimento de matéria prima na quantidade e qualidade necessárias para o processo produtivo, através do controle total que uma organização tem sobre uma atividade em consequência da internalização e acompanhamento constante (MAGALHÃES et al., 2011; RAYCIKI, et al., 2016).

As complexidades da cadeia de valor e as incertezas e/ou perturbações ambientais são motivos para a organização reverter a estratégia do outsorcing e aplicar o insourcing das atividades (FOERSTL et al., 2016). Circunstância constatada ao identificar que o insourcing pode direcionar a melhores custos de transação, variação de demanda, motivação do poder de mercado, entre outros fatores (BENMEHAIA; BRABEZ, 2016).

É possível observar que determinados artigos ressaltam os pontos frágeis da terceirização antes de abordar o insourcing (desterceirização), como por exemplo, o lead time e a variabilidade de qualidade que empresas terceiras podem provocar no processo (CAPUTO; PALUMBO, 2005).

Ainda, pode-se observar que o insourcing foi utilizado como uma solução estratégica durante as crises econômicas, como maneira de mitigar a ociosidade, as capacidades subutilizadas da organização principal (DRAUZ, 2013). Fato corroborado ao mencionar a viabilidade do insourcing diante de custos operacionais menores que o custo do mercado externo (BESANKO et al., 2018). Quanto à análise do nível de evidência, a grande maioria dos artigos achados sinalizam estudos do nível IV, o que revela ausência de pesquisas com abordagens e desenhos metodológicos diversificados, que ampliem o conhecimento acerca deste objeto de estudo que é ainda incipiente.

Os autores dos artigos são profissionais de diversas áreas do conhecimento, tais como engenheiros, administradores de empresa e profissionais da tecnologia da informação. O que indica que a estratégia do insourcing tem sido estudada por diversos profissionais, bem como a aplicabilidade do insourcing tem sido aplicada em variadas organizações: automobilística, têxtil e da computação (CAPUTO; PALUMBO, 2005; QU et al. 2010; DRAUZ, 2013; FOERSTL at al., 2016; HARTMAN et al., 2016; HARTMAN et al., 2017).

Todos os estudos desta $\mathrm{Rl}$ foram conexos no sentido de recomendarem a avaliação do insourcing nas organizações antes de adotarem a estratégia do Outsorcing (desterceirização), pois aquela em determinadas situações podem ser mais vantajosas sob vários aspectos: custo, qualidade do produto, redução de prazos de entrega, melhoria das competências internas, dentre outras. Conhecer todos os parâmetros internos, bem como a dinâmica interna do parceiro possibilita 
uma tomada de decisão adequada a médio e longo prazo (CAPUTO; PALUMBO, 2005; QU et al. 2010; DRAUZ, 2013; FOERSTL at al., 2016; HARTMAN et al., 2016; HARTMAN et al., 2017).

Ações evidenciadas nesta RI, propõe que a administração da empresa deve realizar uma análise da situação do negócio, posição de mercado atual, juntamente com uma descrição clara de suas atividades atuais e de longo prazo. Conforme aponta o estudo de Almeida e Cardoso (2014), isso pode ser integrado por um mapeamento detalhado dos aspectos que resumem os fatores estratégicos de uma empresa utilizando a matriz SWOT, que identifica pontos fortes, pontos fracos, oportunidades e ameaças como ferramenta de identificação do posicionamento da organização em seu ambiente.

No que concerne a área da tecnologia da informação, o valor do insourcing deve ser utilizado para melhorar o desempenho interno, os autores ensejam que as organizações devem considerar a $\mathrm{Tl}$ como parte integrante do seu núcleo estratégico e envolver-se proativamente no desenvolvimento interno dos recursos de T.I (QU et al., 2010).

\section{CONCLUSÃO}

A reconsideração do insourcing tem sido utilizada como uma estratégia organizacional impulsionada por diversos motivos: melhoria do controle das atividades principais; redução dos custos operacionais; melhoria da qualidade; complexidade da cadeia de valor; incertezas ambientais e políticas; modelos de fabricação mais eficientes; supressão da dependência de fornecedores externos e preservação do valor estratégico da organização.

$\mathrm{Na}$ presente revisão integrativa da literatura, a síntese dos resultados dos artigos facilitou a compreensão de evidências, ou seja, acelerou a transferência de conhecimento para a prática. Os achados foram de profissionais de diversos campos, como: Engenharias, Administração e Tecnologia da Informação. As maiores prevalências foram de artigos que fizeram uso do estudo de caso, que consiste na análise de modo detalhado de um caso individual que explica a dinâmica do caso.

Com este procedimento é possível a aquisição de conhecimento do fenômeno estudado a partir da exploração intensa de um único caso. Os achados indicam que o Insourcing nas organizações ainda requer uma aplicação em pesquisa que explore todo seu potencial.

Entretanto, uma lacuna na produção de conhecimento foi revelada ao verificar-se escassez da publicação e indexação em base de dados, tendo em vista que no período de janeiro de 2005 a maio de 2018, foram selecionados seis artigos que atenderam aos critérios de inclusão.

Sugere-se que pesquisas futuras possam dar continuidade na investigação da pratica do insourcing nas organizações para que o conhecimento seja ainda mais difundido para toda comunidade cientifica.

\section{REFERÊNCIAS}

AGUEZZOUL, A. N. Third-party logistics selection problem: A literature review on criteria and methods; Omega 49 69-78; Science Direct - Elsevier. 2014. Disponível em: <https://doi.org/10.1016/j.omega.2014.05.009>. DOI: 10.1016/j.omega.2014.05.009. 
ALMEIDA, A. C. L.; CARDOSO, A. J. G. Diagnóstico Rápido Participativo e Matriz Swot: Estratégias de Planejamento Estratégico com Base na Atual Posição do Curso de Secretariado Executivo UEPA. Revista de Gestão e Secretariado - GeSec, São Paulo, v. 5, n. 2, p 117-137, mai./ago. 2014. Disponível em: <https://www.revistagesec.org.br/secretariado/article/view/283/pdf_57>. DOI: 10.7769/gesec.v5i2.283.

BAETA, O. V.; BRITO, M. J.; SOUZA, R. B. Strategy as Discursive Practice in a Brazilian Public University: A Look under the Perspective of Critical Discourse Analysis. Public Administration Research, v. 3, n. 2, p. 17-27, 2014. Disponível em: < http://dx.doi.org/10.5539/par.v3n2p17>. DOI: 10.5539/par.v3n2p17.

BESANKO, D.; DRANOVE, D.; SHANLEY, M.; SHAEFER, S. A economia da estratégia. 5 ed. São Paulo: Editora. Bookman, 2018.

BENMEHAIA, A. M.; BRABEZ, F. The Empirical Factors Determining Vertical Integration in Algerian Food Manufacturing Sector. European Scientific Journal, ESJ, $\quad$ v. $12, \quad$ n. 28, 2016. Disponível em: <http://dx.doi.org/10.19044/esj.2016.v12n28p\%25p>.

DOI: 10.19044/esj.2016.v12n28p252

CAPUTO, A. C.; PALUMBO, M. Manufacturing re-insourcing in the textile industry: A case study. Industrial management \& data systems, Bingley, v. 105, n. 2, p.193207, 2005. Disponível em: <https://doi.org/10.1108/02635570510583325>. DOI: $10.1108 / 02635570510583325$.

DRAUZ, R. Re-insourcing as a manufacturing-strategic option during a crisis: cases from the automobile industry. Journal of Business Research, v. 67, p. 346-353, 2013. Disponível em: <https://doi.org/10.1016/j.jbusres.2013.01.004>. DOI:10.1016/j.jbusres.2013.01.004.

FOERSTL, K.; KIRCHOFF, J. F.; BALS, L. Reshoring and insourcing: drivers and future research directions. International Journal of Physical Distribution \& Logistics Management, v. 46, n.5, p. 492-515, 2016. Disponível em: <https://doi.org/10.1108/IJPDLM-02-2015-0045>.DOI: $10.1108 /$ IJPDLM-02-20150045.

FONTENELLE, I. A. O estatuto do consumo na compreensão da lógica e das mutações do capitalismo. Lua Nova, n. 92, p. 207-240, 2014. Disponível em: <http://dx.doi.org/10.1590/S0102-64452014000200008>. DOI: 10.1590/S0102.

FREYTAG, Per V.; CLARKE, Ann H.; EVALD, Majbritt R. Reconsidering outsourcing solutions. European Management Journal, v. 30, n. 2, p. 99-110, 2012. Disponível em: <https://doi.org/10.1016/j.emj.2011.11.002>. DOI: 10.1016/j.emj.2011.11.002.

GIOSA, L. A. Terceirização: uma abordagem estratégica. 5.ed. São Paulo: Pioneira Thomson Learning, 2003. 
HARTMAN, P. L.; OGDEN, J. A.; WIRTHLIN, J. R.; HAZEN, B. T. Nearshoring, reshoring, and insourcing: Moving beyond the total cost of ownership conversation. Business Horizons, v. 60, n. 3, p. 363-373, 2017. Disponível em: <https://doi.org/10.1016/j.bushor.2017.01.008>. DOI: 10.1016/j.bushor.2017.01.008.

HARTMAN, P. L.; OGDEN, J. A.; HAZEN, B. T. Bring it back? An examination of the insourcing decision. International Journal of Physical Distribution \& Logistics Management, v. 47, n. 2/3, p. 198-221, 2016. <https://doi.org/10.1108/IJPDLM-092015-0220>. DOI: 10.1108/JJPDLM-09-2015-0220.

MAGALHÃES, Y. T.; CARVALHO NETO, A. M.; SARAIVA, L. A. S. Práticas gerenciais relacionadas à qualificação de trabalhadores terceirizados: um estudo de caso no setor de mineração. Organizações \& Sociedade, Salvador, v. 18, n. 57, p. 227-244, abr.jjun. 2011.2 Disponível em: $<$ https://rigs.ufba.br/index.php/revistaoes/article/view/11147/8059>.

MENDES K. D. S.; SILVEIRA R. C. C. P.; GALVÃO C. M. Revisão integrativa: método de pesquisa para incorporação de evidências na saúde e na enfermagem. Texto \& Contexto-Enfermagem, v. 17, n. 4, p. 758-764, 2008. Disponível em: < http://www.producao.usp.br/bitstream/handle/BDPI/3509/art_MENDES_Revisao_inte grativa_metodo_de_pesquisa_para_a_2008.pdf?sequence=1\&isAllowed=y>.

PORTER, M. Ventaja competitiva: creación y sostenimiento de un desempeño superior. 2. ed. México: Grupo Editorial Patria, 2015.

QU, W. G.; OH, W.; PINSONNEAULT, A. The strategic value of IT insourcing: an ITenabled business process perspective. The Journal of Strategic Information Systems, v. 19, n. 2, p. 96-108, 2010. Disponível em: <https://doi.org/10.1016/j.jsis.2010.05.002>. DOI: 10.1016/j.jsis.2010.05.002.

RAYCIKI, C.; EYERKAUFER, M. L.; MARIAN, S. Terceirização ou primarização: impactos no desempenho econômico e financeiro de uma empresa de serviços especializados. In: Anais do Congresso Brasileiro de Custos-ABC, 2016, Porto de Galinhas. Anais... Porto de Galinhas, PE, 2016.

SARAIVA, L. A. S.; MERCÊS, R. E. Terceirização na gestão da manutenção: estudo de caso de uma mineradora. Revista de Administração, v. 11, n. 1, p. 124, 2013. Disponível em: < http://dx.doi.org/10.15600/rau.v11i1.389>. DOI: 10.15600/1679-5350/rau.v11n1p1-24

SCHNIEDERJANS, M. J.; SCHNIEDERJANS, A. M.; SCHNIEDERJANS, D. G. Outsourcing and insourcing in an international context. 1. ed. New York: Routledge, 2015.

STETLER, C. B. et al. Utilization-focused integrative reviews in a nursing service. Applied Nursing Research, v. 11, n. 4, p. 195-206, 1998. Disponível em: <https://doi.org/10.1016/S0897-1897(98)80329-7>. $\quad$ DOI: $\quad 10.1016 /$ S08971897(98)80329-7. 
URSI, E. S. Prevenção de lesões de pele no pré-operatório: revisão integrativa da literatura. Ribeirão Preto (São Paulo): 2005. 130 f. (Mestrado em Enfermagem) Escola de Enfermagem de Ribeirão Preto da Universidade de São Paulo. 